\title{
Anomalous diffusion and environment-induced quantum decoherence
}

\author{
Luca Bonci, ${ }^{1}$ Paolo Grigolini, ${ }^{1,2,3}$ Adam Laux, ${ }^{1,4}$ and Roberto Roncaglia ${ }^{1}$ \\ ${ }^{1}$ Dipartimento di Fisica dell'Università di Pisa, Piazza Torricelli 2, 56127 Pisa, Italy \\ ${ }^{2}$ Istituto di Biofisica del Consiglio Nazionale delle Ricerche, Via San Lorenzo 26, 56127 Pisa, Italy \\ ${ }^{3}$ Department of Physics of the University of North Texas, P.O. Box 5368, Denton, Texas 76203 \\ ${ }^{4}$ Department of Solid State Physics, Loránd Eötvös University of Sciences, Muzeum krt. 6-8, Budapest 1088, Hungary
}

(Received 21 November 1995)

\begin{abstract}
We study the anomalous diffusion resulting from the standard map in the so-called accelerating state, and we observe that it is determined by unusually large times of sojourn of the classical trajectories in the fractal region at the border between the chaotic sea and the acceleration island. The quantum-mechanical breakdown of this property implies a coherence among so slightly different values of momentum as to become much more robust against environment fluctuations than the quantum localization corresponding to normal diffusion.
\end{abstract}

[S1050-2947(96)01107-9]

PACS number(s): 03.65.Bz, 05.40.+j, 05.45.+b

\section{INTRODUCTION}

In quantum mechanics the superposition of two independent states, both referring to a physically plausible solution of the Schrödinger equation, is still a plausible solution of the Schrödinger equation. If the two solutions have a classical meaning, for instance, they are two distinct trajectories, it is not clear how to interpret their superposition, which, yet, according to quantum mechanics should be regarded as a valid picture of reality. A widely accepted solution of this problem is afforded by the decoherence theory (DT) recently made quite popular by Zurek [1] but corresponding to a viewpoint shared by many other authors [2]. It rests on the assumption that there are no such things in nature as isolated systems. Thus if a macroscopic oscillator, with mass $M$ and friction $\Gamma$, is prepared in a superposition, $c_{+}|+\rangle+c_{-}|-\rangle$, of two states, $|+\rangle$ and $|-\rangle$, corresponding to two distinct positions separated by the distance $\Delta Q$, as an effect of the environmental fluctuations the coherence between these two states is lost within a time defined by

$$
t_{\mathrm{dec}}=\frac{\hbar^{2}}{2 M \sigma(\Delta Q)^{2}},
$$

with $\sigma=\Gamma k_{B} T$. Notice that this decoherence time is proportional to the square of the Planck constant. Thus it turns out to be virtually instantaneous, if the parameters of the oscillator have values corresponding to a macroscopic body yielding for the denominator of (1) an extremely large value. This is the key physical interpretation behind all the approaches resting on the essential role of the environment to recover classical from quantum physics [1-3]. A careful examination of this theoretical approach to the transition from quantum to classical mechanics [4] reveals that it rests on the assumption that the system of interest, as well as the environment, is driven by ordinary equilibrium statistical physics. The main purpose of this paper is to study the consequence of rejecting the assumption that the system undergoes an ordinary process of statistical mechanics, while retaining the plausible assumption that the environment still obeys this condition.

\section{QUANTUM-CLASSICAL CORRESPONDENCE IN ORDINARY STATISTICAL MECHANICS}

Let us now focus our attention on the kicked rotator [5]. In the classical case, this system is described by the standard map

$$
\begin{gathered}
p_{t+1}=p_{t}+K \sin \theta_{t}, \\
\theta_{t+1}=\theta_{t}+p_{t+1} \bmod (2 \pi) .
\end{gathered}
$$

This is an area-preserving map driving the discrete evolution of a classical rotator kicked at regular intervals of time by an impulsive torque with a strength proportional to $K \sin \theta$. In the case of strong chaos $(K \gg 1)$ the variable $K \sin \theta$, as well as $\theta$, can be perceived as a quickly fluctuating variable, resulting in a diffusional process for the momentum $p$. The fluctuating variable $K \sin \theta$ is characterized by a finite-time scale, and in the long-time limit this diffusion process turns out to be Gaussian (on the basis of the central limit theorem) and the second moment $\left\langle p^{2}(t)\right\rangle$ becomes a linear function of time. We shall refer to this process as normal diffusion and as an example of ordinary statistical mechanics.

To discuss the corresponding quantum-mechanical description let us observe the time evolution of the Wigner distribution subsequent to an initial condition with the Wigner distribution virtually identical to the Liouville distribution. This implies the adoption of a Planck constant $\hbar$ much smaller than the volume of the classical phase space explored by the system during the process of observation. Note that, as usual in the field of quantum chaos, the "Planck constant"' we adopt is actually a parameter expressing the ratio of the characteristic classical action to the real Planck constant. Zurek and Paz [6] show that as a result of the process of fragmentation of the Liouville distribution, the quantum corrections to the classical Poisson brackets (classical term) become important at the time

$$
t_{\chi}=\frac{1}{\lambda} \ln \left(\frac{\chi}{\hbar}\right),
$$

where $\lambda$ is the Lyapunov coefficient of the classical and chaotic trajectories and $\chi$ is a scale parameter proportional to 
the derivative of the potential of order higher than the second. This is a widely accepted property in the field of quantum chaos [7] and implies that, in spite of the fact that the true value of the Planck constant is extremely small, the transition from classical to quantum physics, in a condition where the correspondence principle is expected to apply, might take place at times compatible with an experimental observation.

However in the case of the standard map no significant statistical discrepancy between quantum and classical time evolution is found at that time. We think that this is subtly related to the reasons why strong chaos makes the linearresponse theory work, in spite of the fact that the fragmented Liouville density responds nonlinearly to the influence of external perturbations. If the fragmented distribution is used to weight a smooth variable the result of the averaging can turn out to be independent of the details of the fragmentation [8]. Thus the essential equivalence between quantum and classical mechanics seems to be due to the statistical equivalence of quantum and classical distribution, in spite of significant discrepancies of their fine details. A different but probably equivalent way of accounting for this property rests on the remark that normal diffusion, in classical physics, is a statistical process insensitive to the presence of weak fluctuations [9].

In conclusion, as widely stressed in [10], a fair discussion of the correspondence between quantum and classical mechanics implies that the former is compared with classical statistical mechanics. This is so because a quantummechanical mean value implies that the observation process results in the collapse of the wave function into the eigenstates of the observable under study. This is a stochastic process implying the adoption of a Gibbs picture, and therefore making quite natural the adoption of the Wigner formalism which immediately establishes a comparison between quantum mechanics and the classical Liouville equation. As pointed out in [4], the classical statistical processes used so far to establish a correspondence between quantum and classical physics pertain to the field of ordinary statistical processes, for instance, ordinary Brownian motion.

In the standard map the discrepancy between classical and quantum physics is made evident at later times by the occurrence of the well-known phenomenon of quantum localization (QL) [5]. As pointed out by many authors [11], the process of normal diffusion is broken after a time, the localization time $t_{L}$, of the order

$$
t_{L} \propto \frac{K^{2}}{\hbar^{2}}
$$

and is replaced by a process where $\left\langle p^{2}(t)\right\rangle$ fluctuates around a constant value. This conflict between classical and quantum mechanics, albeit occurring at astronomical large times in the case of macroscopic systems, has been cured by many authors [12] adopting essentially the perspective that there are no systems in nature which are isolated from the environment. Thus the quantum-mechanical counterpart of a system such as

$$
\begin{gathered}
p_{t+1}=p_{t}+K \sin \theta_{t}+f_{t}, \\
\theta_{t+1}=\theta_{t}+p_{t+1} \bmod (2 \pi)
\end{gathered}
$$

was considered, where $f_{t}$ is a white Gaussian noise of variance $\sigma$. The discussion of how to derive this white noise from a rigorous Hamiltonian coupling with the environment is the object of an intense research effort $[12,13]$. This whitenoise approximation was derived within a rigorously Hamiltonian treatment by Cohen and Fishman [13] in the hightemperature limit. We are convinced that the simulation of the nonlocal quantum interaction between a system of interest and its quantum environment might conflict with this white-noise assumption, especially in the case of weak environment fluctuations. This is related with the intriguing problem of the validity of the Markov approximation which has been escaping us for years, and still is, a satisfactory settlement. However, since the Markov approximation is a widely adopted assumption, and the related white-noise assumption is widely used by many researchers $[6,12,13]$, we accept both of them here with no discussion. An important reason for this choice, in addition to the fact that it is universally made, is that many experimental processes, for instance in the field of quantum optics, are satisfactorily explained by theories resting on the Markov approximation, and even if this is not theoretically well founded, it cannot be ruled out on the basis of a conflict with experimental data. In this paper we prefer to focus our attention on the robustness of the quantum fluctuations against white-noise fluctuations, because this has physical consequences which are in principle experimentally observable. The recent analysis of Shiokawa and Hu [14] shows that these methods, resting on the picture of Eq. (5), are essentially equivalent to the DT. Furthermore, these authors find the following expression for the decoherence time [to compare to (1)]:

$$
t_{\mathrm{dec}}=\frac{\hbar^{2}}{2 \sigma(\Delta p)^{2}},
$$

where $\Delta p=\ell \hbar=K^{2} / \hbar, \ell$ being the so-called localization length. Again, as in (1), the denominator in (6) refers to macroscopic quantities thereby resulting in a decoherence time much shorter than the localization time (4), and consequently in the breakdown of localization with noises of relatively small intensity.

\section{NUMERICAL RESULTS: ANOMALOUS VERSUS NORMAL DIFFUSION}

This section is devoted to the discussion of the numerical results illustrated by Figs. 1 and 2. The numerical results concerning the classical case are obtained via numerical integration of the equation of motion (5). As to the quantum version of it, this is obtained by building up the quantum mapping corresponding to (5). This is realized by applying to the wave function $\left|\psi^{t}\right\rangle$ the Floquet operator $F$, so that

$$
\left|\psi^{t+1}\right\rangle=F\left|\psi^{t}\right\rangle .
$$

The explicit form of the Floquet operator corresponding to the classical map of (5) is

$$
F=e^{-(i / 2 \hbar)\left(p+f_{t}\right)^{2}} e^{-(i / \hbar) k \cos q} .
$$

We establish the initial condition in the quantum case by setting the wave function in the eigenstate of the momentum 

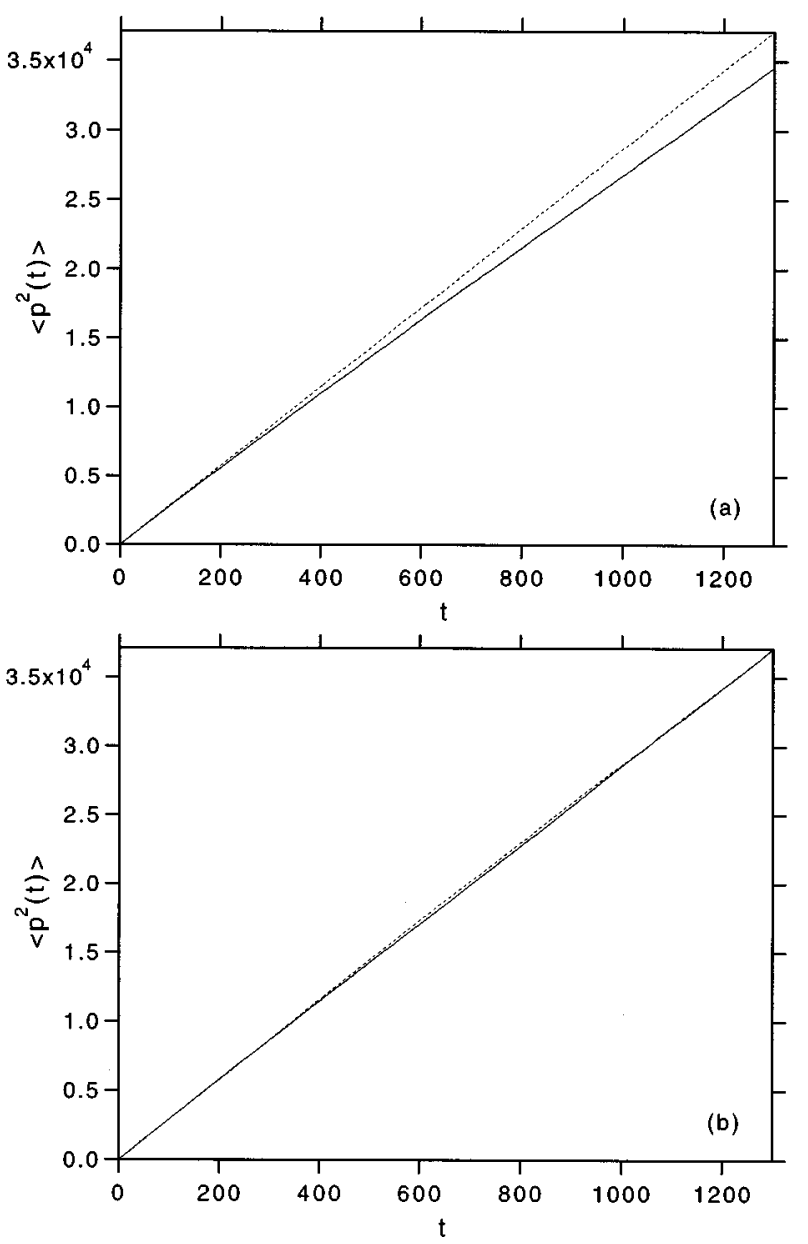

FIG. 1. The mean value $\left\langle p^{2}(t)\right\rangle$ as a function of time. All the curves refer to $K=6$, a value of the control parameter implying, in classical physics, normal diffusion. The dashed curves denote the classical case and the solid lines refer to the quantum case. Figure 1(a) refers to the case of the kicked rotator isolated from its environment. Figure 1(b) refers to the case of the kicked rotator interacting with the environment according to Eq. (5), classical case, and Eqs. (7) and (8), quantum case. The noise intensity is $\sigma=4 \times 10^{-6}$ and the value of the Planck constant adopted is $\hbar=0.01$

with a vanishing eigenvalue. In the classical case, to make a fair comparison between the classical and the quantum prediction, we distribute the angle variable uniformly between 0 and $2 \pi$. As a consequence of this choice the early time evolution predicted by quantum mechanics is made to coincide with the classical time evolution so as to fit the main purpose of this paper, namely, the discussion of the quantum-classical correspondence, as the assessment of the time span of the statistical equivalence between quantum and classical dynamics: this is guaranteed in the early part of the dynamical process by a wise choice of the initial conditions and it is eventually broken at later times by the emergent quantum correlations.

Figure 1 shows that, according to the DT, external fluctuations play an efficient role to recover classical from quantum mechanics. The comparison between quantum and classical time evolution of the second moment $\left\langle p^{2}(t)\right\rangle$, with no influence of external fluctuations, is made in Fig. 1(a). We
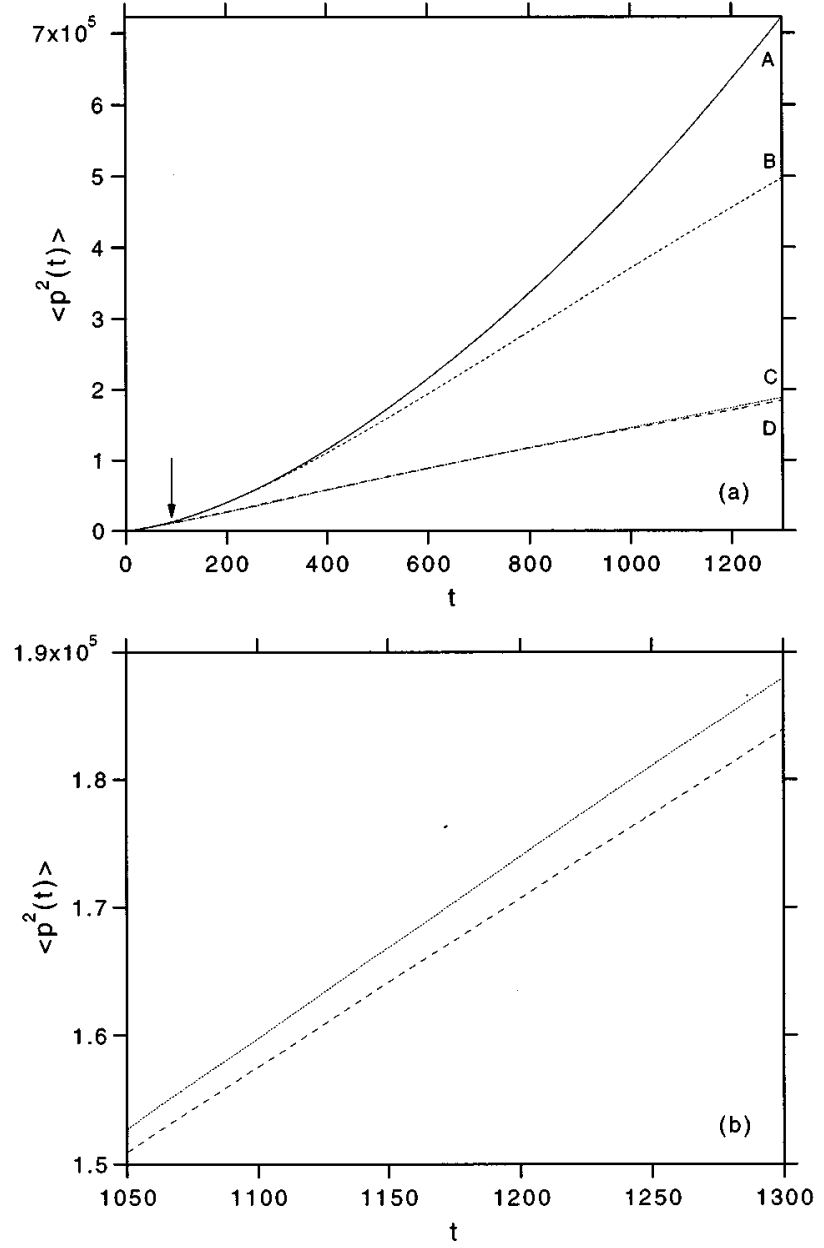

FIG. 2. The mean value $\left\langle p^{2}(t)\right\rangle$ as a function of time. All the curves refer to $K=6.9115$, a value of the control parameter implying, in classical physics, anomalous diffusion. The two upper curves of Fig. 2(a) illustrate the classical case, without external noise (A) and with external noise (B). The two lower curves of Fig. 2(a) denote the quantum case, without external noise (D) and with external noise (C). The arrow in Fig. 2(a) denotes the time at which, according to (13), see [10], QBAD occurs. Figure 2(b) shows the curves $\mathrm{C}$ and $\mathrm{D}$ of Fig. 2(a) in a more enlarged scale. Note that the dotted line refers to the quantum case with external noise while the dashed line corresponds to the quantum case with no external noise. The noise intensity is $\sigma=4 \times 10^{-6}$ and the value of the Planck constant adopted is $\hbar=0.01$. Notice that these are the same values as those used in the case normal diffusion illustrated in Fig. 1.

see that the quantum dynamics show a clear sign of the localization process, under the form of an increasing departure of the quantum prediction (solid line) from the classical (dashed line). The comparison between the quantum and the classical case in the presence of weak external fluctuations is illustrated by Fig. 1(b): we see that the quantum case (still denoted by the solid line) is virtually indistinguishable from the classical case (still denoted by the dashed line). Notice that the attainment of the equivalence between quantum and classical dynamics implies only a slight change of the classical time evolution of $\left\langle p^{2}(t)\right\rangle$. By inspection of Fig. 1, it is seen that the dashed curve of Fig. 1(a) is virtually coincident with the dashed curve of Fig. 1(b). This is a consequence of the fact that the noise intensity chosen, $\sigma=4 \times 10^{-6}$, is very 
weak. In the quantum case, on the contrary, as weak as this external noise is, its intensity is large enough as to produce the significant change from the solid line of Fig. 1(a) to the solid line of Fig. 1(b), a change virtually identical to that from the solid line of Fig. 1(a) to the dashed line of Fig. 1(a): this confirms that the DT is a satisfactory way to establish a correspondence between quantum and classical mechanics in the case of ordinary (classical) diffusion.

The kicked rotator also affords an attractive example of dynamics corresponding to nonordinary statistical mechanics. It has to do with the special values of the control parameter $K$ (we use the value $K=6.9115$ ) corresponding to the birth of accelerating islands, which produce a momentum diffusion faster than ordinary Brownian motion, with $\left\langle p^{2}(t)\right\rangle$ increasing faster than linearly in time. All this is widely discussed in Refs. $[15,16]$ and it is illustrated here by the upper solid curve (denoted by the letter A) in Fig. 2(a), which clearly shows a deviation of $\left\langle p^{2}(t)\right\rangle$ from the linear dependence on time.

The quantum-mechanical dynamics corresponding to this condition was studied in Ref. [10]. It was shown that the use of small but finite values of $\hbar$ leads to an early time evolution of the mean-squared momentum which is indistinguishable from the predictions of classical mechanics. In Fig. 2(a) this corresponds to the time region with the upper limit denoted by the arrow: after this time the quantum case, denoted by the dashed line labeled by $\mathrm{D}$, departs from the classical dynamics. This departure time $t_{\mathrm{QB}}$ is expected to be an increasing function of $1 / \hbar$.

We refer to this as the phenomenon of quantum breakdown of anomalous diffusion (QBAD). In Ref. [10] arguments are given to support the view that $\mathrm{QBAD}$ time $t_{\mathrm{QB}}$ is a function of $\hbar$ with the same logarithmic structure as in (3). On an intuitive ground this is related to the fact that the time at which the Wigner distribution departs from the Liouville density now has a direct effect on diffusion, since it sets an upper time limit to the sojourn of the "trajectories" in the region at the border between chaotic sea and accelerating islands [10] (see also Sec. IV).

We see from Fig. 2(a) that this unusual statisticalmechanical condition leads to the failure of the DT as an interpretative scheme to recover classical from quantum mechanics. In the classical case the presence of a fluctuation as intense as that studied in Fig. 1(b) makes the anomalous diffusion behavior described by curve A of Fig. 2(a) change into the normal diffusion described by curve $\mathrm{B}$. This shows that after a given time $t_{\mathrm{CB}}$ the process of diffusion becomes normal again. This phenomenon is well understood theoretically and was studied in Refs. [9,17]. On the basis of the results illustrated in Fig. 1 one would expect that a noise of the same intensity should make the quantum result coincide with the classical. We see, on the contrary, that this noise does not significantly affect the quantum result: curve $\mathrm{C}$ of Fig. 2(a), taking into account the influence of noise, is essentially indistinguishable, on the scale of the figure, from curve $\mathrm{D}$ denoting the quantum evolution with no environmental influence.

The response of the quantum system to the external fluctuation is illustrated in an enlarged view in Fig. 2(b), which shows that actually the two quantum results depart one from the other. The long-time linear behavior of the quantum re- sult with noise (upper curve) is not followed by the quantum evolution without noise, the slope of which becomes increasingly smaller upon increase of time, in a qualitative agreement with the departure of the two corresponding curves in Fig. 1(a). The order of magnitude of the two effects is the same and we think that both of them reflect the same process: the well-known QL phenomenon [5,11].

We want to point out that the decoherence time (6), expressing the standard prescription of DT [14], evaluated by choosing as $\Delta p$ the overall dimension of the wave function at the time $t_{\mathrm{QB}}$ would result, for the case of Fig. 2(a), in a time smaller than one time step, thereby implying the quantum prediction to coincide with the classical. This conflicts with our numerical results, showing that the quantum case, denoted by curve $\mathrm{C}$, is clearly distinct from the corresponding classical case, denoted by curve B. This suggests that, in the case of anomalous diffusion, a more careful evaluation of $\Delta p$, expressing the $p$ size of the quantum coherence, has to be made. This is discussed in Sec. IV.

In conclusion these results suggest this qualitative picture: quantum fluctuations provoke a transition from anomalous to ordinary diffusion, and this "ordinary" diffusion, due to the quantum nature of the system, is expected to undergo a process of localization at later times. Environmental fluctuations, with an intensity large enough as to break QL do not affect QBAD, in spite of the fact that the coherence responsible for QL is destroyed by environmental fluctuations in times much shorter than the QBAD time. This means that the quantum coherence behind $\mathrm{QBAD}$ is more robust against environmental fluctuations than the quantum coherence responsible for QL.

\section{THEORETICAL INSIGHTS AND CONCLUSIONS}

This section is devoted to accounting for the results of Sec. III theoretically, with arguments that are expected to become more and more reliable with decreasing the value of the Planck constant and eventually exceeding the limited range of the current computer treatments [10]. First of all, we have to shortly review the key ingredients behind anomalous diffusion in classical physics. In the specific case of the kicked rotator, anomalous diffusion is caused by the fact that the value $K=6.9115$ of the control parameter used for the case of Fig. 2(a), corresponds to the presence of accelerating islands imbedded in the chaotic sea of the $(p, \theta)$ phase space. The border between the chaotic sea and one accelerating island, called a laminar region, is fractal and self-similar $[15,16]$, and for this reason the corresponding distribution of sojourn times $\Psi(t)$ for a particle moving in this region, is shown, adopting a Markovian hierarchy of zones of quasistability [18], to have the inverse power-law structure

$$
\lim _{t \rightarrow \infty} \Psi(t) \propto \frac{1}{t^{\mu}},
$$

with the index $\mu$ found numerically [15] and analytically [19] to fulfill the condition:

$$
2<\mu<3 .
$$


It is shown $[20,21]$ that the second moment of $p$ must have in the long-time limit the following behavior:

$$
\left\langle p^{2}(t)\right\rangle=H t^{4-\mu},
$$

where $H$ denotes a constant factor. Under condition (10) this leads to the anomalous diffusion of Fig. 2(a) (curve A). Note that the case studied numerically in the present paper refers to $\mu=2.667$.

The theoretical model used to account for the inverse power law (9) is based on the tree model of [19]: at each step the system branches in either a "transversal" motion, essential to derive the correct value of the index $\mu$, or in a "longitudinal" motion leading the trajectories, more quickly than the "transversal," to explore regions of the phase space of so small size as to be incompatible with the dynamics of a quantum distribution. This longitudinal motion is characterized by the scaling parameters $\delta$ and $\beta$, for times and areas, respectively. This means that while the trajectory imbeds itself more and more deeply in the laminar region, the size of the region explored becomes smaller and smaller $(\beta<1)$ and the motion becomes slower and slower $(\delta<1)$. The joint action of the transversal and the longitudinal motion results in a value of the index $\mu$ fitting very satisfactorily the numerical calculations [19].

Let us now discuss the quantum case. Let us denote by $\Xi(0)$ the area of the "doorway" portions of the laminar region, those directly arrived at by trajectories starting from the chaotic sea. The early part of the diffusion process [the times preceding the arrow of Fig. 2(a)] coincides with the classical prediction. To account for this property we must assume that $\hbar$ is smaller than $\Xi(0)$. It is interesting to notice that the classical trajectories traveling through the laminar regions are not regular, but chaotic, since they are associated with small but finite Lyapunov coefficients [22]. As pointed out in [10,23], as small as it is, a finite Lyapunov coefficient implies the exponential increase of the quantum uncertainty $U(t)=\Delta p(t) \Delta q(t)$. At the same time the size of the region explored by the quantum distribution is a known decreasing function of time $\Xi(t)$. Note that on the basis of geometrical information on the phase space [22] we are led to assume that $\Delta \theta$ is of the same order of magnitude as $\Delta p$ (for simplicity we set $\Delta \theta=\Delta p$ ). Thus we can derive expressions of $\Delta p$ as a function of $\Xi(t)$, to use later to quantitatively estimate the robustness of the new effect.

We maintain that the motion of the quantum distribution departs from that of the classical distribution at the time $t=t_{\mathrm{QB}}$ defined by the condition

$$
U(t)=\Xi(t) .
$$

Thus, the theoretical model adopted, in addition to accounting for the power-law nature of the waiting time distribution (9), leads us [10] to predict that anomalous diffusion is broken as an effect of quantum fluctuations, and normal diffusion is recovered, at the time $t_{\mathrm{QB}}$ given by

$$
t_{\mathrm{QB}} \propto \frac{1}{\lambda_{f}} \ln \frac{1}{\hbar},
$$

where $\lambda_{f}$ denotes the Lyapunov coefficient of the laminar region. Notice that this prediction has the same structure as that by Zurek and Paz [6]. However, it is now adapted to a condition where the microscopic departure of the quantum from the classical distribution, implying the breakdown itself of the concept of classical trajectory, has an immediate statistical consequence: the inverse power-law distribution of waiting times (9) is truncated and the resulting diffusion process, due to the special sensitivity of anomalous diffusion to fluctuations [9], is made normal again.

We need now to establish the minimum size of the quantum coherence established in the fractal region by the wave packet expressed in the $p$ representation. This will allow us to determine the corresponding decoherence time according to the prescriptions of the DT. Using scaling arguments on the Markov model of Ref. [18] we find the size of the phasespace structures reached at the QBAD time. From this we get

$$
\Delta p=\left(\Xi(0) /\{\ln [\Xi(0) / \hbar]\}^{\gamma}\right)^{1 / 2},
$$

where $\gamma=\left(\ln \beta \delta^{2} / \ln \beta \delta\right)(\ln \beta / \ln \delta)$. Let us evaluate the decoherence time (6) by assigning to $\Delta p$ the value (14). This results in a decoherence time larger than the QBAD time, thereby accounting for the result of Fig. 2(a).

Notice that anomalous diffusion is destroyed by environmental fluctuations also in the classical case. By setting the condition that the process of motion through the laminar region is stopped when regions of the same size as the noise variance $\sigma$ are reached, the time $t_{\mathrm{CB}}$ of crossover from anomalous to normal diffusion is shown to be

$$
t_{\mathrm{CB}} \propto \sigma^{-(\ln \delta / \ln \beta)} .
$$

We find this prediction to be in a remarkably good agreement with our numerical results: using the tree model we have $\ln \delta / \ln \beta=0.48$ and the numerical determination of $t_{\mathrm{CB}}$ results in the power index 0.46 . The result of (15), compared to the logarithmic prediction (13), suggests the striking observation that quantum-mechanical fluctuations can break anomalous diffusion earlier than thermal fluctuations.

Let us come back to the quantum case. If the same assumption is made as that by Lai, Ott, and Grebogi [24], that the quantum distribution is only stopped by structures as large as $\hbar$ itself, and the exponential increase of $U(t)$ is disregarded, we obtain

$$
t_{\mathrm{QB}} \propto \hbar^{-(\ln \delta / \ln \beta)}
$$

and

$$
\Delta p=\hbar^{(1 / 2)} .
$$

The comparison of (16) with (15) points out the "classicallike" nature of the assumption by Lai, Ott, and Grebogi [24]: notice that the computer results of [10] seem to support our conviction that the prediction (13) reflects more properly the effects of quantum mechanical coherence.

To assess more firmly that the quantum manifestation corresponding to the QBAD effect is more robust against environmental fluctuations than the traditional QL effect, we estimate

$$
R_{N} \equiv \frac{t_{\mathrm{dec}}}{t_{L}} \quad \text { and } \quad R_{A} \equiv \frac{t_{\mathrm{dec}}}{t_{\mathrm{QB}}} .
$$


The estimate of $R_{A}$ can either be made adopting the prescription of Lai, Ott, and Grebogi [24] [based on (6), (16), and (17)] or that of Roncaglia et al. [10] [based on (6), (13), and (14)]. We denote the corresponding estimates of $R_{A}$ by $R_{1}$ and $R_{2}$, respectively. In the limit of small values of $\hbar$, we get

$$
R_{N} \propto \hbar^{6} \ll R_{1} \propto \hbar^{1.5}, \quad R_{N} \ll R_{2} \propto \hbar^{2}\left(\ln \frac{\Xi(0)}{\hbar}\right)^{\gamma-1},
$$

which establishes QBAD as more robust than QL, whatever prediction, either (13) or (16), is adopted. For this reason QBAD seems to be an interesting channel for the macroscopic manifestation of quantum mechanics to be contrasted to that proposed by Leggett [25]. The more plausible logarithmic breakdown (13) is compatible with the direct observation of the QBAD effect even in the classical limit of small but finite $\hbar$ 's. However according to the DT this implies the noise intensity to be so weak as to realize the condition $R_{A}>1$. Is this possible? Answering this question implies further theoretical research work and the design of experiments along the promising lines established by the authors of Refs. [26]. In these experiments the parameter $\hbar$ is an "effective Planck constant" determined by the experimental conditions according to the prescriptions of Refs. [26]. To make the results of this research work more relevant to the discussion of the correspondence principle, the value of this parameter should be so small as to make completely negligible quantum-mechanical effects such as the tunneling from the chaotic sea into the island and back. The value of $\hbar$ defines the area of a portion of the phase space which should be set much smaller than the area of the fractal region surrounding the accelerating islands. In our numerical treatment $(k=6.9115)$ the area of the overall region, including both accelerating island and the fractal region around it, is of the order of 1. On the other hand, the size of the fractal region is about one order of magnitude smaller than the area of the phase space pertaining to the accelerating islands [22]. Therefore the value $\hbar=0.01$, used in Fig. 1, cannot yet be regarded as being much smaller than the area of the fractal region so as to realize a fully satisfactory classical condition. However from the dynamical data of Fig. 1 we see that the early part of the dynamical process is indistinguishable from the prediction of classical physics. This means that the tunnel effect, if any exists, is of moderate intensity.

To establish whether the QBAD time obeys the powerlaw prediction (16) or the logarithmic prediction (13) we would need to study the dynamics corresponding to still smaller values of $\hbar$, and this, at the moment, conflicts with the limitations of our computer facilities. We think, however, that this paper provides conclusive evidence, supported by both numerical results and theoretical arguments, that the quantum-mechanical breakdown of anomalous diffusion is much more robust against external fluctuations than the process of quantum localization.

The result of this paper might have remarkable consequences on the foundation of quantum mechanics, if further numerical, or, hopefully, experimental evidence, will support our conviction that the time $t_{\mathrm{QB}}$ has a logarithmic dependence on $\hbar$. This would be equivalent to making quantum effects observable also in the classical limit, in spite of the presence of environmental fluctuations. Although the DT is a rigorous application of quantum mechanics, its failure to recover classical from quantum physics would not automatically imply that quantum mechanics is wrong. We cannot rule out the possibility that the results of this paper disclose a new avenue to quantum manifestations in the classical regime, and that a real experiment might prove the existence of the quantum-mechanical effects that we predict. On the other hand, if quantum mechanics will be proved to be correct again, the failure of the DT would imply that the problem of how to recover classical from quantum physics is not yet settled, and doubts would be cast on the possibility that this can be done from within quantum mechanics.
[1] W.H. Zurek, Phys. Today 44(10), 36 (1991).

[2] P. Grigolini, Quantum Irreversibility and Measurement (World Scientific, Singapore, 1993).

[3] W.H. Zurek, Phys. Rev. D 24, 1516 (1981); D 26, 1862 (1982); E. Joos and H.D. Zeh, Z. Phys. B 59, 223 (1985); A.O. Caldeira and A.J. Leggett, Phys. Rev. A 31, 1059 (1985); W.G. Unruh and W.H. Zurek, Phys. Rev. D 40, 1071 (1989); B.L. Hu, J.P. Paz, and Y. Zhang, Phys. Rev. D 45, 2843 (1992); Phys. Rev. D 47, 1576 (1993); W.H. Zurek, Prog. Theor. Phys. 89, 281 (1993).

[4] P. Grigolini, in Chaos-The Interplay Between Stochastic and Deterministic Behaviour, Vol. 457 of Lecture Notes in Physics, edited by P. Garbaczewski, M. Wolf, and A. Weron (Springer-Verlag, Berlin, 1995).

[5] F.G. Casati, B.V. Chirikov, F.M. Izrailev, and J. Ford, in Stochastic Behavior in Classical an Quantum Hamiltonian Systems, Vol. 93 of Lecture Notes in Physics, edited by F.G. Casati and J. Ford (Springer-Verlag, Berlin, 1979).

[6] W.H. Zurek and J.P. Paz, Phys. Rev. Lett. 72, 2508 (1994).
[7] M.V. Berry, Ann. Phys. (N.Y.) 131, 163 (1981); G.M. Zaslavsky, Phys. Rep. 80, 157 (1981).

[8] M. Bianucci, R. Mannella, B.J. West, and P. Grigolini, Phys. Rev. E 50, 2630 (1994).

[9] R. Bettin, R. Mannella, B.J. West, and P. Grigolini, Phys. Rev. E 51, 212 (1995).

[10] R. Roncaglia, L. Bonci, B.J. West, and P. Grigolini, Phys. Rev. E 51, 5524 (1995).

[11] F.M. Izrailev, Phys. Rep. 196, 299 (1990), and references therein.

[12] E. Ott, T.M. Antonsen, Jr. and J.D. Hanson, Phys. Rev. Lett. 53, 2187 (1984); T. Dittrich and R. Graham, Phys. Rev. A 42, 4647 (1990); D. Cohen, ibid. 43, 639 (1991); D. Cohen, Phys. Rev. Lett. 67, 1945 (1991).

[13] D. Cohen and S. Fishman, Phys. Rev. A 39, 6478 (1989).

[14] K. Shiokawa and B.L. Hu, Phys. Rev. E 52, 2497 (1995).

[15] T. Horita, H. Hata, R. Ishizaki, and H. Mori, Prog. Theor. Phys. 83, 1065 (1990); R. Ishizaki, H. Hata, T. Horita, and H. Mori, Prog. Theor. Phys. 84, 179 (1990). 
[16] J. Klafter, G. Zumofen, and M.F. Shlesinger, Europhys. Lett. 25, 565 (1994).

[17] E. Floriani, R. Mannella, and P. Grigolini, Phys. Rev. E 52, 5910 (1995).

[18] J.D. Hanson, J.R. Cary, and J.D. Meiss, J. Stat. Phys. 39, 27 (1985).

[19] J. D. Meiss and E. Ott, Phys. Rev. Lett. 55, 2741 (1985); Physica D 20, 387 (1986).

[20] G. Zumofen and J. Klafter, Phys. Rev. E 47, 851 (1993).

[21] G.Trefan, E. Floriani, B.J. West, and P. Grigolini, Phys. Rev. E 50, 2564 (1994).

[22] J. Klafter, G. Zumofen, and M.F. Shlesinger, in Chaos-The Interplay Between Stochastic and Deterministic Behaviour, Vol. 457 of Lecture Notes in Physics, edited by P. Garbac- zewski, M. Wolf, and A. Weron (Springer-Verlag, Berlin, 1995).

[23] L. Bonci, R. Roncaglia, B.J. West, and P. Grigolini, Phys. Rev. Lett. 67, 2593 (1991); Phys. Rev. A 45, 8490 (1992).

[24] Y. Lai, E. Ott, and C. Grebogi, Phys. Lett. A 173, 148 (1993).

[25] A.J. Legget, in Chance and Matter, Proceedings of the Les Houches Summer School, Les Houches, 1986, edited by J. Souletie, J. Vannimenus, and R. Stora (North-Holland, Amsterdam, 1987).

[26] F.L. Moore, J.C. Robinson, C. Bharucha, P.E. Williams, and M.G. Raizen, Phys. Rev. Lett. 73, 2974 (1994); J.C. Robinson, C. Bharucha, F.L. Moore, R. Jahnke, G.A. Georgakis, Q. Niu, M.G. Raizen, and B. Sundaram, Phys. Rev. Lett. 74, 3963 (1995). 